\title{
Ambulatory Assessment of Balance
}

\author{
H. Martin Schepers, Student Member, IEEE, Edwin H.F. van Asseldonk, Jaap H. Buurke, \\ Bart F.J.M. Koopman, and Peter H. Veltink, Member, IEEE
}

\begin{abstract}
Gait analysis is commonly done in gait laboratories, where several gait variables are estimated using measurement systems installed in the laboratory. Two important variables to characterize human gait are the Center of Mass (CoM) and the Center of Pressure (CoP). The major drawback of existing measurement systems is their restriction to the laboratory. This study demonstrates the possibilities of an ambulatory measurement system: the forceshoe. It consists of an orthopaedic sandal with six-degrees-of-freedom force/moment sensors beneath the heel and the forefoot and an inertial sensor rigidly attached to each force/moment sensor. For validation, the measurement system has been compared to a reference system consisting of an optical position measurement system and two force plates. The root-mean-square $(\mathrm{rms})$ difference between the CoP trajectories was $(0.0051 \pm 0.0007) \mathbf{m}$, corresponding to $(1.7 \pm 0.3) \%$ of the length of the shoe. The rms difference between the CoM trajectories estimated by both measurement systems was $(0.025 \pm 0.007) \mathbf{m}$. Based on the results presented in this study, it is concluded that the instrumented shoe allows accurate and continuous estimation of gait variables that can be used to assess balance.
\end{abstract}

\section{INTRODUCTION}

Human walking is often linked to the motion of two coupled pendula. The double support phase is viewed as a transition from one inverted pendulum to the next. The pendulum model is characterized by two variables: the Center of Mass (CoM) and the Center of Pressure (CoP). The CoM is an imaginary point at which the total body mass can be assumed to be concentrated. The $\mathrm{CoP}$ is the application point of the ground reaction force, the point on the contact surface between body and ground where the moments about the horizontal axes are zero. An efficient means to stabilize this essentially unstable system is to adjust foot placement [1].

Traditionally, CoM and CoP are estimated in so-called 'gait laboratories', equipped with optical position measurement systems for body movement and force plates for the ground reaction force. However, a major drawback of these measurement systems is the restriction in their use to these laboratories. Therefore research is required for the development of measurement systems to perform these measurements in an ambulatory environment.

This study was financially supported by ZonMW (grant number: 1435.0026) and the Dutch Ministry of Economic Affairs under the FreeMotion project.

H.M. Schepers, E.H.F van Asseldonnk H.F.J.M Koopman and P.H. Veltink are with the Institute for Biomedical Technology (BMTI), University of Twente, P.O. Box 217, 7500 AE Enschede, The Netherlands. (e-mail: h.m.schepers@ewi.utwente.nl).

Jaap H. Buurke is with Roessingh Research and Development, 7522 AH Enschede, The Netherlands. He is also with Roessingh Rehabilitation Center, 7522 AH Enschede, The Netherlands.
Besides the aforementioned restriction to the laboratory environment, the measurement of the ground reaction force using a force plate has some other disadvantages. First, the subjects are required to place their feet completely on the force plates in order to perform a correct force measurement. Since the subjects should not adapt their natural gait pattern, it can be rather time consuming to acquire correct force measurements. Second, only one or two steps can be measured during a trial, so many successive trials are usually required. Finally, it is impossible to distinguish the ground reaction force acting on each foot when standing with both feet on a single plate, as only the total ground reaction force is registered.

Measuring body movement by an optical position measurement system offers accurate position tracking tracking of body segments. However, the line of sight from camera to marker is often blocked by the subject, resulting in incomplete data. An alternative to these optical position measurement systems is to use inertial (accelerometers and gyroscopes) and magnetic sensors [2]-[4]. Although these sensors do not suffer from the drawbacks of the optical measurement systems, these are not ideal as well. The estimation of position and orientation requires integration of acceleration and angular velocity respectively, which gives rise to inherent drift caused by noise and a fluctuating offset. However, this drift can be avoided by the use of suitable estimation algorithms [5]-[8].

Several methods exist for CoM estimation, of which the segmental kinematics method [9] and the double integration of ground reaction force method [10], [11] are the most important ones. The segmental kinematics method is based on the definition of the CoM and models the body as a kinematic chain of rigid segments. By measuring the position and orientation of each segment and by approximating the mass fractions as well as the locations of the CoM of each segment, an estimation of the overall CoM movement is obtained. The double integration of ground reaction force method is based on Newton's second law, which states that the net external force acting upon a body is equal to its mass multiplied by its acceleration. Since the external force and body mass can be measured accurately by for example a force plate, an estimation of the acceleration of the CoM is obtained. The displacement of the CoM can be calculated through double integration of acceleration after subtracting gravitational acceleration, with proper consideration of initial constants of integration, i.e. initial velocity and position. Although widely accepted [12], [13], these methods have their limitations as well. The performance of the segmental 


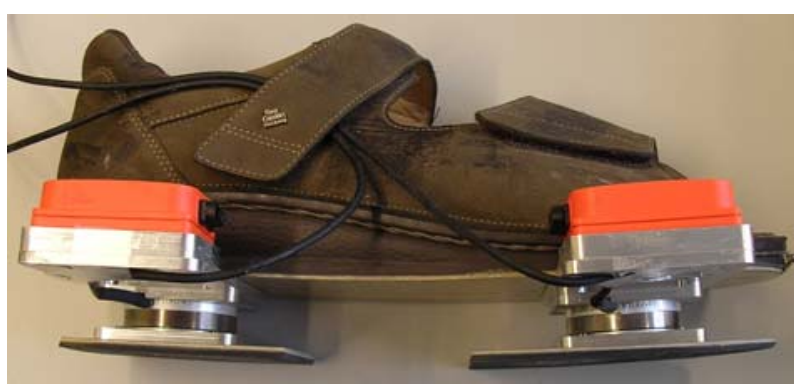

Fig. 1. Picture of the instrumented shoe with force/moment sensors beneath the heel and forefoot and inertial sensors rigidly attached to the force/moment sensors.

kinematics method depends heavily on the accuracy of the approximated segmental mass fractions and segment CoM locations [14]. Moreover, it requires precise marker or sensor placement for accurate position and orientation determination. The performance of the double integration of ground reaction force method is limited by the accuracy of the determined initial constants of integration. A joint drawback is the restriction to a laboratory environment and the accompanying inability for continuous measurement of the CoM trajectory.

The objective of this study is to show the ability of the forceshoe to estimate variables used for balance assessment as an alternative to the traditional systems restricted to a laboratory. The forceshoe (Fig. 1) is an ambulatory measurement system consisting of an orthopaedic sandal equipped with force/moment sensors and inertial sensors able to measure the ground reaction force and movement of foot and ankle [15], [16]. The forceshoe is used to estimate CoP and CoM movement continuously. The system is validated using a force plate and an optical position measurement system as a reference.

\section{Methods}

\section{A. Estimation of Center of Pressure Trajectory}

The CoP is defined as the application point of the ground reaction force, the point on the contact surface between body and ground where the moments about the horizontal axes are zero. Since the 3D force, 3D moment and the movement of the force/moment sensors are estimated, the location of the CoP can be extracted. A detailed description of the method to extract the CoP can be found in [16].

\section{B. Estimation of Center of Mass Trajectory}

The estimation of CoM movement is based on fusion of CoP data with double integrated ground reaction force data, both estimated from signals measured by the forceshoe. The fusion is based on a frequency domain method, which is described extensively in [17].

\section{Experimental Methods}

To compare the accuracy of the ambulatory measurement system with a conventional measurement system, experiments were performed in a gait laboratory, where an optical tracking system (Vicon Oxford Metrics, Oxford, UK) was available. Subjects wearing instrumented shoes and inertial sensors were instructed to walk repeatedly through the laboratory between predefined start and end points. Body movement and ground reaction force were measured by the instrumented shoes and inertial sensors, while the optical tracking system was used as a reference. The instrumented shoes consisted of standard orthopaedic sandals equipped with two six-degrees-of-freedom force/moment sensors (ATIMini45-SI-580-20, supplier: Schunk, Arnhem, NL) beneath the heel and forefoot, as shown in Fig. 1. The weight of each shoe was $1.1 \mathrm{~kg}$. Each force sensor had a miniature inertial sensor (Xsens Motion Technologies, Enschede, The Netherlands) rigidly attached to it, for the estimation of position and orientation. For the reference system, markers were placed on both legs (toe, heel, lateral malleolus, shank, knee, thigh), both arms (upper arm, elbow, head of ulna and styloid process of radius), left and right anterior superior iliac spine, left and right shoulder, and a 3 cluster marker on the sacrum. The CoM displacement using the reference system was determined by the segmental kinematics method, based on the model of Koopman et al. [18].

Data from the inertial sensors, the analogue data from the force sensors beneath the shoe and the 3D marker data were sampled at $50 \mathrm{~Hz}$. All data were low-pass filtered by applying a second order recursive Butterworth filter, at a cutoff frequency of $15 \mathrm{~Hz}$. The voltages from the force sensors were converted to forces by applying calibration matrices. The synchronization between the inertial sensor system and Vicon was done by maximizing the correlation between pulses generated by a pulse generator that were sent to both measurement systems. Possible gaps in the Vicon data were spline-interpolated prior to filtering with a maximum gapsize of 15 samples.

\section{REsults}

An estimation of the three components of the ground reaction force is shown in Fig. 2. The axes of the global coordinate system are defined by positive $x$ in the direction of gait, positive $z$ in the upward direction, and positive $y$ perpendicular to the $x$ and $z$ direction such that the result is an orthogonal right-handed coordinate system. It should be noted that the forces are normalized with respect to body weight. The signals measured with the forceshoe (black) shows good correspondence with the signals measured with the force plate (gray), which is confirmed by the rootmean-square (rms) difference between the magnitudes of the ground reaction force, being $(0.012 \pm 0.001) \mathrm{N} / \mathrm{N}$ (mean \pm standard deviation), being $(1.1 \pm 0.1) \%$ of the maximal magnitude. The CoP estimated by both measurement systems is shown in Fig. 3. The trajectories agree well, resulting in a rms difference between both methods of $(0.0051 \pm 0.0007)$ $\mathrm{m}$, corresponding to $(1.7 \pm 0.3) \%$ of the length of the shoe. The rms difference between the magnitudes of the position estimates using both methods (Fig. 4) was calculated as $(0.018 \pm 0.006) \mathrm{m}$, being $(1.4 \pm 0.5) \%$ of the maximal magnitude. 


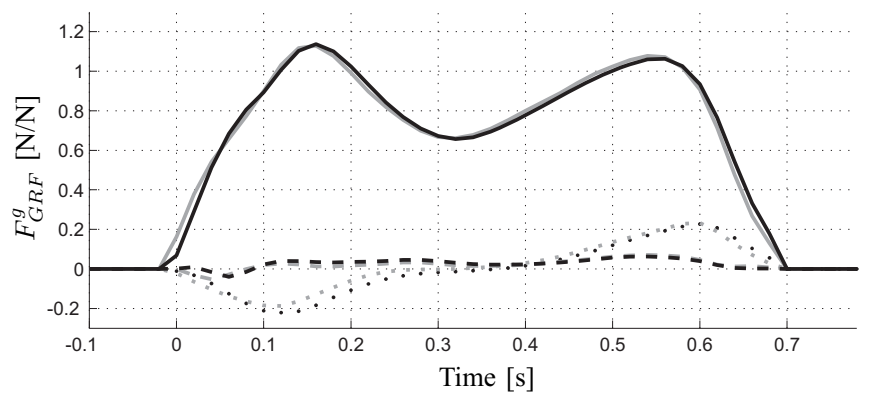

Fig. 2. Three components of the ground reaction force (x: dotted; $y$ : dashed; z: solid) measured by the instrumented shoe (black) and the force plate (gray).

The ability of the forceshoe to measure several steps during a single measurement is shown in Fig. 5. The figure shows an integration of the measured ground reaction force (vertical lines) with the estimated position of the heel (dots). The ability to measure several steps is also shown in Fig. 6, which shows the estimated CoM estimated by the ambulatory (solid) and reference (dashed) systems. On either side of the $\mathrm{CoM}$, the CoP is indicated by the black dots where each dot represents a time sample. The limited measurement volume of the reference system is shown by the gray area. The $x, y$ and $z$ components of the CoM trajectory are shown in Fig. 7. It should be noted that the integrated mean velocity has been subtracted from the $x$ component to visualize differences

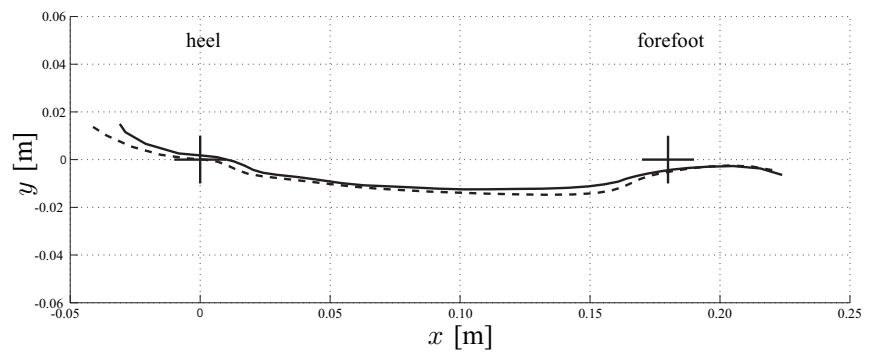

Fig. 3. CoP trajectory estimated using ambulatory (solid) and reference (dashed) systems. The black crosses indicate the center of each force sensor.

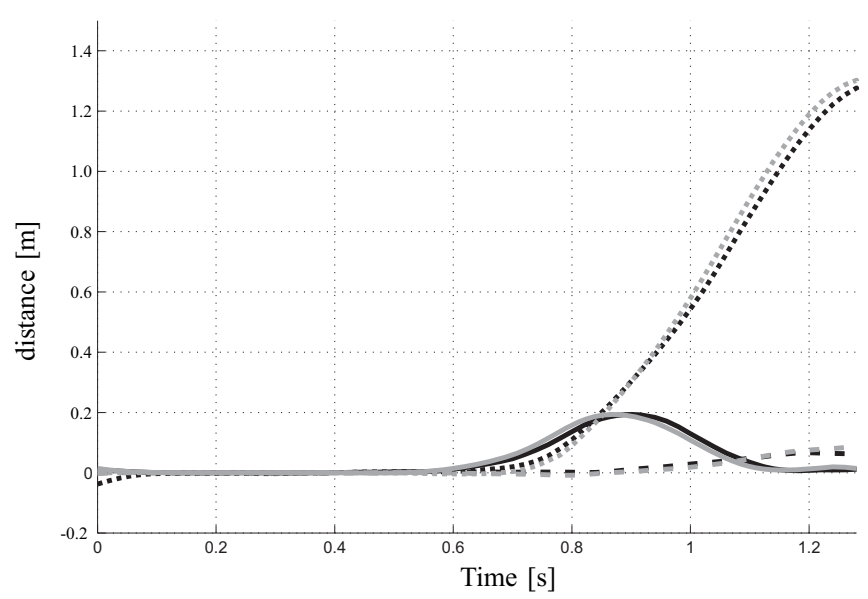

Fig. 4. 3D position (x: dotted, $y$ : dashed, z: solid) of the heel force sensor estimated by the instrumented shoe (black) and Vicon (gray).

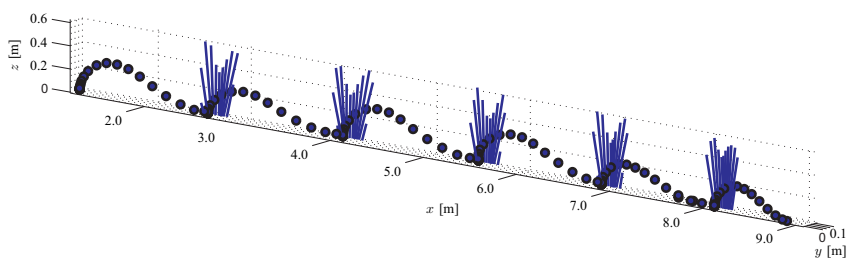

Fig. 5. Estimation of the ground reaction force (vertical lines) and the movement of the heel (dots) of the right foot during several steps.

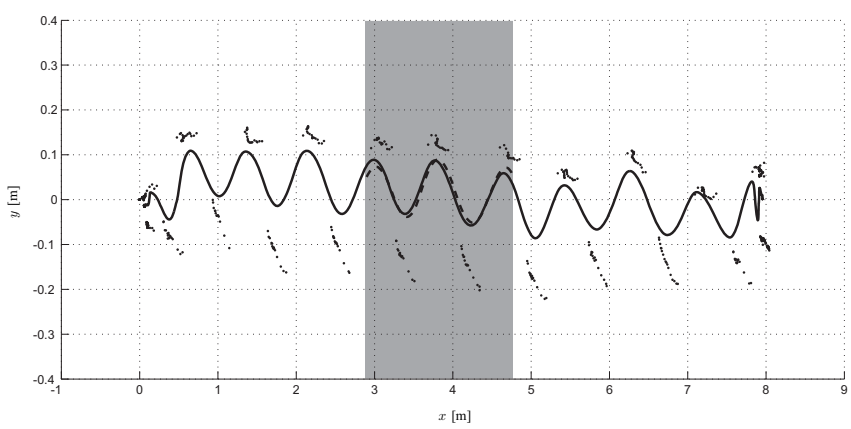

Fig. 6. Estimated CoM by the ambulatory (solid) and reference (dashed) systems. The CoP is indicated by the black dots on either side of the $\mathrm{CoM}$, where each dot represents a time sample. Moreover, the measurement volume of the reference system is indicated by the gray area.

between both measurement systems in that direction. The rms difference between the magnitudes of the CoM displacement estimated by the ambulatory (solid) and the reference (dashed) measurement systems was $(0.025 \pm 0.007) \mathrm{m}$.

\section{DISCUSSION}

Continuous estimation of gait variables to assess balance, using the ambulatory measurement system described in this study, has several advantages compared to existing measurement systems. The ambulatory measurement system allows continuous estimation $\mathrm{CoP}$ as well as CoM movement during everyday life, whereas existing measurement systems are
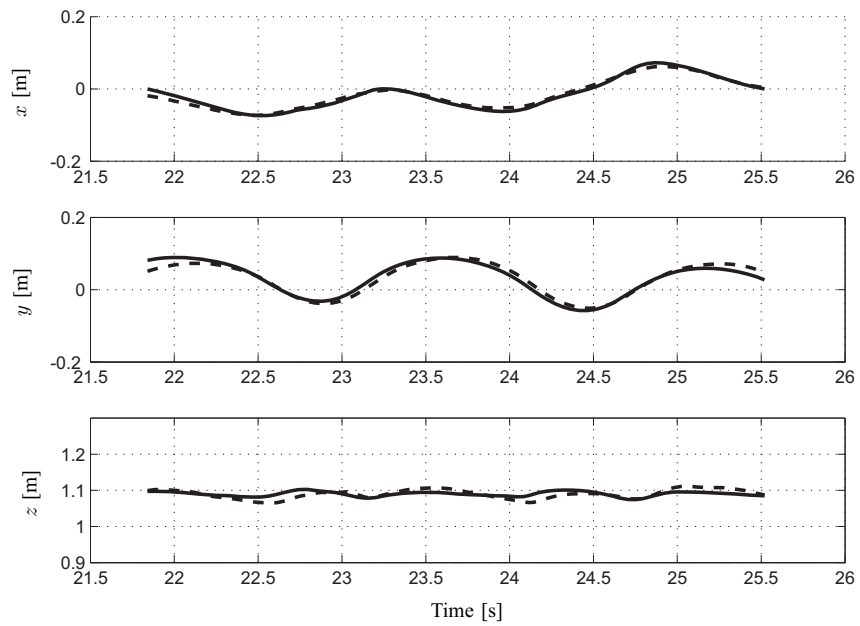

Fig. 7. Three components of the CoM (x: upper; y: middle; z: lower) as a function of time estimated by the ambulatory (solid) and reference (dashed) systems. The integrated mean velocity has been subtracted from the $x$ component. 
restricted to the laboratory and have a limited measurement volume. Especially for pathological gait, continuous estimation is important, since their walking pattern can be variable and asymmetrical. Another advantage of the proposed measurement system is that the measurements can be performed merely wearing the instrumented shoes.

Currently, the instrumented shoe is rather heavy and bulky as shown in Fig. 1. Nevertheless, the influence of the shoe on the gait pattern appeared to be small as reported by Liedtke et al. [19]. In that study, an evaluation of several gait parameters was performed with healthy subjects wearing the instrumented shoes, light, normal and heavy weight shoes. Significant differences between the shoe types were found in maximum ground reaction force only, but these differences could not be attributed to individual shoe types. Moreover, the differences were small compared to the body weight of the subjects and were therefore not considered relevant. The small influence on gait was confirmed by the experience of patients, who were able to walk comfortably with the instrumented shoes. The adapted gait was registered by both the ambulatory and the reference systems and the differences appeared to be small. The purpose of this study was the introduction of the measurement method instead of optimizing the shoe design. In principle, a design optimization will result in a reduction of the influence on gait, while the accuracy of the measurement system remains unaffected.

\section{REFERENCES}

[1] M. A. Townsend, "Biped Gait Stabilization Via Foot Placement," Journal of Biomechanics, vol. 18, no. 1, pp. 21-38, 1985.

[2] D. Roetenberg, "Inertial and magnetic sensing of human motion," $\mathrm{PhD}$ Thesis, University of Twente, 2006.

[3] H. J. Luinge, "Inertial sensing of human movement," Ph.D. dissertation, University of Twente, 2002.

[4] E. R. Bachmann, "Inertial and magnetic tracking of limb segment orientation for inserting humans into synthetic environments," $\mathrm{PhD}$ Thesis, Naval Postgraduate School, 2000.

[5] H. M. Schepers and P. H. Veltink, "Ambulatory estimation of foot movement during gait using inertial sensors," in First Dutch Conference on Bio-Medical Engineering, Egmond aan Zee, the Netherlands, 2007, p. 127.
[6] D. Roetenberg, H. J. Luinge, C. T. M. Baten, and P. H. Veltink, "Compensation of magnetic disturbances improves inertial and magnetic sensing of human body segment orientation," IEEE Transaction on Neural Systems and Rehabilitation Engineering, vol. 13, no. 3, pp. 395-405, 2005.

[7] H. J. Luinge and P. H. Veltink, "Measuring orientation of human body segments using miniature gyroscopes and accelerometers," Medical and Biological Engineering and Computing, vol. 43, no. 2, pp. 273$282,2005$.

[8] X. Yun and E. R. Bachmann, "Design, implementation, and experimental results of a quaternion-based kalman filter for human body motion tracking," Robotics, IEEE Transactions on [see also Robotics and Automation, IEEE Transactions on], vol. 22, no. 6, pp. 12161227,2006

[9] D. A. Winter, Biomechanics and Motor Control of Human Movement. New York: Wiley, 1990.

[10] G. A. Cavagna, "Force platforms as ergometers," J Appl Physiol, vol. 39 , no. 1 , pp. 174-179, 1975.

[11] T. Shimba, "An estimation of center of gravity from force platform data," J Biomech, vol. 17, no. 1, pp. 53-60, 1984.

[12] M. H. A. Eames, A. Cosgrove, and R. Baker, "Comparing methods of estimating the total body centre of mass in three-dimensions in normal and pathological gaits," Hum Mov Sci, vol. 18, no. 5, pp. 637-646, 1999.

[13] E. M. Gutierrez-Farewik, A. Bartonek, and H. Saraste, "Comparison and evaluation of two common methods to measure center of mass displacement in three dimensions during gait," Hum Mov Sci, vol. 25, no. 2, pp. 238-256, 2006.

[14] D. Lenzi, A. Cappello, and L. Chiari, "Influence of body segment parameters and modeling assumptions on the estimate of center of mass trajectory," J Biomech, vol. 36, no. 9, pp. 1335-1341, 2003.

[15] P. H. Veltink, C. B. Liedtke, E. Droog, and H. van der Kooij, "Ambulatory measurement of ground reaction forces," IEEE Trans Neural Syst Rehabil Eng, vol. 13, no. 3, pp. 423-427, 2005.

[16] H. M. Schepers, H. F. J. M. Koopman, and P. H. Veltink, "Ambulatory assessment of ankle and foot dynamics," IEEE Trans Biomed Eng, vol. 54, no. 5, pp. 895-902, 2007.

[17] H. M. Schepers, E. H. F. van Asseldonk, J. H. Buurke, and P. H Veltink, "Ambulatory estimation of center of mass displacement during walking," IEEE Transactions on Neural Systems and Rehabilitation Engineering, vol. Submitted, 2008.

[18] H. F. J. M. Koopman, H. J. Grootenboer, and H. J. de Jongh, "An inverse dynamics model for the analysis, reconstruction and prediction of bipedal walking," J Biomech, vol. 28, no. 11, pp. 1369-1376, 1995.

[19] C. B. Liedtke, S. A. W. Fokkenrood, J. T. Menger, H. van der Kooij, and P. H. Veltink, "Evaluation of instrumented shoes for ambulatory assessment of ground reaction forces," Gait Posture, vol. 26, pp. 3947, 2007. 